\title{
Strategy Towards Active Food Packaging Material From Cellulose Nanoparticles and its Characterization
}

\author{
Ganesan Anusiya ${ }^{1}$, AlaguThirumurugan ${ }^{1, *} \mathbb{D}$, Thyagarajan Sathishkumar ${ }^{1}$, Kuppamuthu Kumaresan ${ }^{1}$, \\ Rengaraj Jaiganesh ${ }^{2}$ \\ 1 Department of Biotechnology, Kumaraguru College of Technology, Coimbatore-641049, Tamilnadu, India \\ 2 Department of Biotechnology, School of Bioengineering, SRM Institute of Science and Technology, Kattankulathur, \\ Chennai - 603203, Tamilnadu, India \\ * Correspondence: biotechthiru@gmail.com, thirumurugan.a.bt@kct.ac.in (A.T.);
}

Scopus Author ID 57001188700

Received: 29.08.2021; Revised: 16.10.2021; Accepted: 20.10.2021; Published: 19.12.2021

\begin{abstract}
The utilization of agro-industrial wastes such as sugarcane bagasse (SCB) as a source of cellulose has influenced a wide range of interest in various applications such as food packaging, drug delivery, paper production, etc. Owing to the rich source of cellulose in SCB, the nanoparticle was prepared efficiently. The pure form of cellulose was isolated from SCB by eliminating the remaining components such as hemicellulose and lignin by treating SCB with a soluble base and a bleaching agent. Cellulose nanoparticles were synthesized from the purified cellulose by acid hydrolysis using $\mathrm{H}_{2} \mathrm{SO}_{4}$ followed by dialysis to remove sulfate ions and attain neutrality. The obtained nanoparticles were characterized using FTIR spectroscopy that helped to confirm the exclusion of lignin and hemicellulose. The crystalline nature of the cellulose nanoparticles (CNPs) was confirmed using X-Ray Diffraction (XRD). The morphology of CNPs was studied by scanning electron microscopy (SEM), and the particle size of CNPs was found to be $189 \mathrm{~nm}$ by particle size analysis (PSA). Further, this study proved the nanomaterial preparation from agro-wastes can be utilized to develop food packaging film in food industries.
\end{abstract}

Keywords: sugarcane bagasse; cellulose nanoparticles; FTIR; XRD; SEM; PSA.

(C) 2021 by the authors. This article is an open-access article distributed under the terms and conditions of the Creative Commons Attribution (CC BY) license (https://creativecommons.org/licenses/by/4.0/).

\section{Introduction}

Sugarcane bagasse, an abundant fibrous agro-industrial waste, is produced in sugar production. In India, about 80 million metric tons (MMT) bagasse are generated, among which 70 MMT are utilized in steam and power industries, while the remaining are left unemployed [1]. The second leading producer and consumer of sugar worldwide is India and is the most important producer of sugarcane after Brazil [1-3]. The constituents of sugarcane bagasse are $40-50 \%$ crystalline cellulose, which is added up with hemicelluloses and xylose, arabinose, galactose, and mannose that are amorphous in structure $[4,5]$. The remaining components in SCB include lignin, mineral, wax, and so on $[6,7]$.

Cellulose is one of the most profuse biodegradable polymers present in nature that is continually restored due to photosynthesis. It is the utmost important component of almost all the plants, including cotton, wood, hemp, cereal straws, sugarcane bagasse, pomaces of fruits and vegetables, and so forth [8]. Nanocellulose can be utilized in several areas, including papers, biocomposites, emulsion and dispersion technology, oil recovery, medical, and cosmetic applications, but the food applications were initially acknowledged as an exceedingly 
captivating application field for nanocellulose because of the rheological nature of the nanocellulose gel [9]. Cellulose nanocrystals are needle-like particles of cellulose that are crystalline in nature with a dimension equal to or lesser than $100 \mathrm{~nm}$ [10].

There are various studies reported on cellulose extraction and nanocellulose preparation from agro-wastes like cassava peel [11], Calotropis procera biomass[12], Potato peel [13], Sesame husk [14], Jack fruit peel [15], pineapple leaf [16], garlic skin [17], and sugarcane bagasse [8], [18, 19]. Though few reports are available on cellulose nanofibres from sugarcane bagasse, a local cultivar was used in this study. Therefore, the primary purpose of the present study is to employ SCB as a source for extraction of cellulose and conversion into nanoparticles, which is an effective strategy towards packing of food materials and their characterization by FTIR, XRD, SEM, and PSA. The characterized nanoparticles can be used to develop food packaging film in future studies.

\section{Materials and Methods}

\subsection{Sample collection.}

SCB, a locally available agro-waste, was collected from a local sugarcane juice shop in Coimbatore, Tamilnadu, India. The collected SCB was cleaned and air-dried for about 1 week. Then, the dried SCB was cut into tiny pieces and then ground into a powder [6].

\subsection{Isolation of cellulose.}

The powdered sample of $5 \mathrm{~g}$ was dewaxed with 1:2(v/v) of ethanol and toluene in a water bath at $75^{\circ} \mathrm{C}$ for 4 hours and then dried. The exclusion of lignin from the dewaxed sample was repeated three times using $1.3 \% \mathrm{NaClO}_{2}$ (adjusted to a $\mathrm{pH}$ range of 3.5 to 4 using $10 \%$ acetic acid) and then dried. The hemicellulose removal was performed by treating the delignified sample with $10 \%$ sodium hydroxide at $25^{\circ} \mathrm{C}$ for 17 hours under continuous stirring $[18,20,21]$. After the expulsion of non-cellulosic components from SCB, cellulose in purified form is obtained, estimated using the anthrone method [22].

\subsection{Synthesis of cellulose nanoparticles.}

The hemicellulose and lignin-free purified cellulose was acid hydrolyzed by refluxing it with $64 \%(\mathrm{w} / \mathrm{w})$ of sulphuric acid in a ratio of $1: 10 \mathrm{~g} / \mathrm{mL}$ under vigorous stirring at $45^{\circ} \mathrm{C}$. After 1 hour, the hydrolysis was quenched by adding 10 folds of distilled water. Eliminating the acidic solution, centrifugation of the sample was performed for 15 minutes at 10,000 rpm, and the supernatant was discarded. Dialysis of the sample was done using distilled water as a solvent for 5-7 days to attain neutrality and exclude sulfate ions [23]. The mixture obtained after dialysis was then homogenized to produce nanocellulose by sonicating for 10 minutes [24].

\subsection{Characterization studies.}

\subsubsection{FTIR analysis.}

An FTIR analysis was performed for the untreated and treated SCB to confirm the elimination of non-cellulosic components using FTIR (Shimadzu, Japan). The spectra were 
taken in the range of $4000-400 \mathrm{~cm}^{-1}$ with a resolution of $4 \mathrm{~cm}^{-1}$, and the reading was observed using \% Transmittance.

\subsubsection{Analysis of crystallinity by XRD.}

An XRD analysis was performed for the obtained CNPs using an X-ray diffractometer (Empyrean, Malvern Panalytical) with $\mathrm{Cu} \mathrm{K \alpha}$ radiation source of wavelength 1.54 A operating at $45 \mathrm{kV}$ voltage and $30 \mathrm{~mA}$ current. The degree of crystallinity of the CNPs was derived using the formula described by Rabek [25]:

$$
R C(\%)=(A c /(A c+A a)) \times 100
$$

where, $A c$ - is the area of crystalline peak and $A a$ is the area of the amorphous peak on the Xray diffractogram.

The average size of the crystals of CNPs was calculated using the following Scherer formula:

$$
\tau=K \lambda /(\beta \cos \theta)
$$

where, $\lambda$ denotes the wavelength of the $\mathrm{X}$-ray used $\left(1.54 \mathrm{~A}^{\circ}\right), \theta$ is the diffraction angle, $\mathrm{K}$ is the shape factor (0.94), and $\beta$ is the full width at half maximum of the peak (FWHM) [26].

\subsubsection{Analysis of morphology by Scanning electron microscopy (SEM).}

The morphological analysis of prepared cellulose nanoparticles from SCB was performed using scanning electron microscopy. SEM micrographs of surfaces were taken using a scanning electron microscope (CAREL ZEISS - EVO 18). The samples were coated with gold by the sputtering technique.

2.4.5. Particle size analysis by dynamic light scattering (DLS).

The particle size of the prepared cellulose nanoparticles was measured by dynamic light scattering (DLS) using Micromeritics, Model: Nano plus. The conditions maintained for measurement: refractive index of water, 1.33 ; viscosity, $0.8878(\mathrm{cP})$; and temperature, $25^{\circ} \mathrm{C}$.

\section{Results and Discussion}

\subsection{Isolation of cellulose and nanoparticles preparation.}

In this work, cellulose was isolated in purified form from SCB, and it was converted into nanocellulose by acid hydrolysis. Removal of wax from SCB before chemical treatment using the mixture of toluene and ethanol leads to a better yield of purified cellulose [27]. After dewaxing the sample, the wax content in the SCB was removed, which was ensured by the reduction in the sample weight from $5 \pm 0.2 \mathrm{~g}$ to $4.5 \pm 0.2 \mathrm{~g}$. Delignification using sodium chlorite is generally a bleaching process, which turns the sample white, indicating the exclusion of lignin. Treating the delignified sample using alkali paves the way for hydrogen bond breakage, resulting in higher hemicellulose removal [28], as shown in Figure 1. Whitening the material post-purification confirms that a great quantity of the non-cellulosic components present in the beginning was eliminated [16]. The yield percentage of purified cellulose was 
found to be $44 \pm 2.3 \%$, and the cellulose concentration in the purified sample was estimated to be $1.5 \mathrm{mg} / \mathrm{mL}$.

After cellulose was prepared, acid hydrolysis was performed to generate cellulose nanoparticles from purified cellulose. Further, to break the sample into linear fragments, it was homogenized using a sonicator. After sonication, the obtained slurry revealed a remarkably high viscosity. A similar observation has been stated by Dos Santos et al. (2013) [16].

Dewaxing with1:2 (v/v)

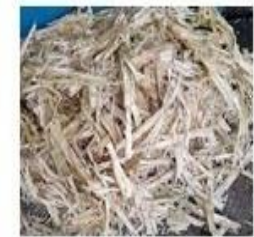

SCB
About 1 week air dried and gound into powder

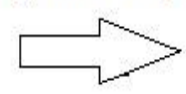

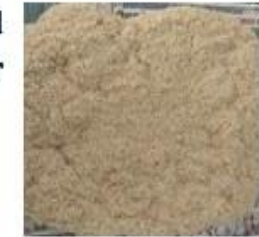

Powdered SCB of ethanol and toluene in a water bath at $75^{\circ} \mathrm{C}$ for 4 hoûrs

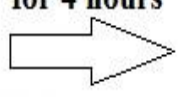

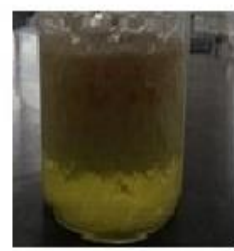

Dewaxed sample

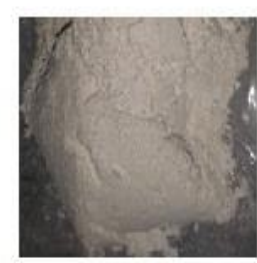

Cellulose nanoparticles
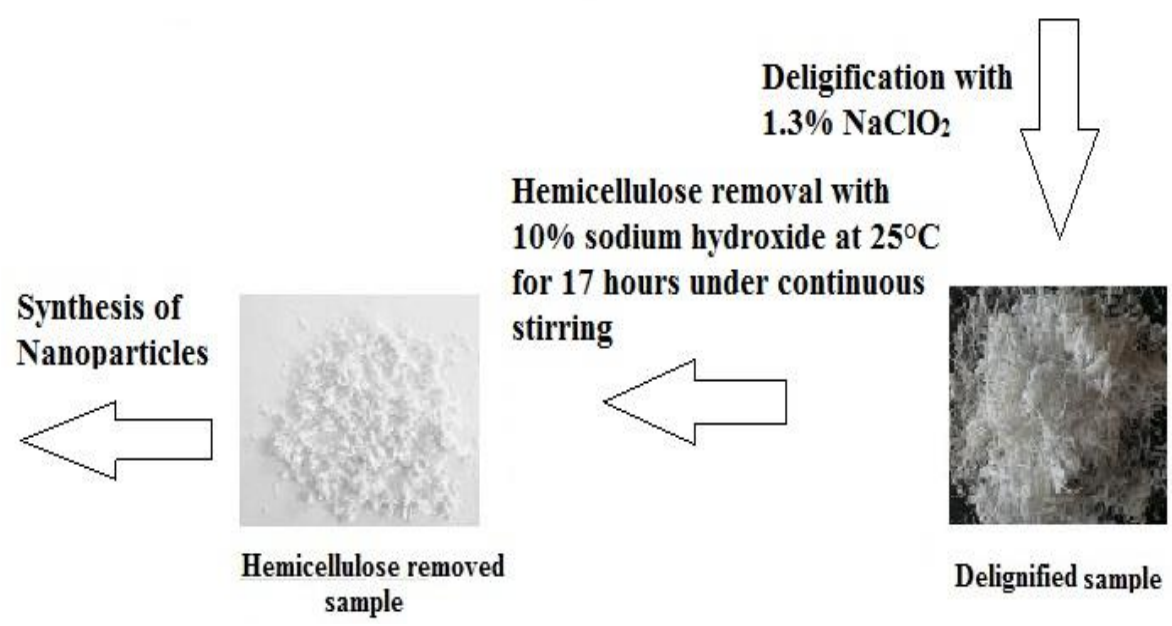

Figure 1. Schematic steps involved in the preparation of CNPs from SCB.

\subsection{Characterization studies.}

3.2.1. Fourier Transform infrared spectroscopy (FTIR).

FTIR has been broadly used in research based on polysaccharides like cellulose because of its accurate prediction of chemical changes that arise after the treatment using the chemical method. The changes that occurred in the structure of SCB were observed by comparing the FTIR spectrum of SCB pre- and post-chemical treatment, as shown in Figure 2. The peaks between 3200 and $3400 \mathrm{~cm}^{-1}$ were because of the $\mathrm{O}-\mathrm{H}$ stretching of the hydrogen-bonded hydroxyl group of lignin and cellulose [29]. The strong peaks at $1726 \mathrm{~cm}^{-1}$ and $1242 \mathrm{~cm}^{-1}$ were observed in untreated SCB, which denotes the acetyl uronic ester groups of hemicelluloses, the ester linkage of the carboxylic group of the ferulic and p-coumaric acids of lignin or hemicelluloses. These peaks were not observed in the cellulose spectra because of the elimination of non-cellulosic compounds by chemical treatments [30], thereby depicting that the cellulose molecular structure remains unaffected even after acid hydrolysis.

3.2.2. X-ray diffraction (XRD).

The XRD was done to examine the crystalline structure of CNP produced by the chemical method. The sample showed a higher peak intensity at about $2 \theta=22.19$, which is 
interrelated to the cellulose crystal structure. The degree of crystallinity and crystal size of CNPs was derived from the X-ray diffractogram (Figure 3).

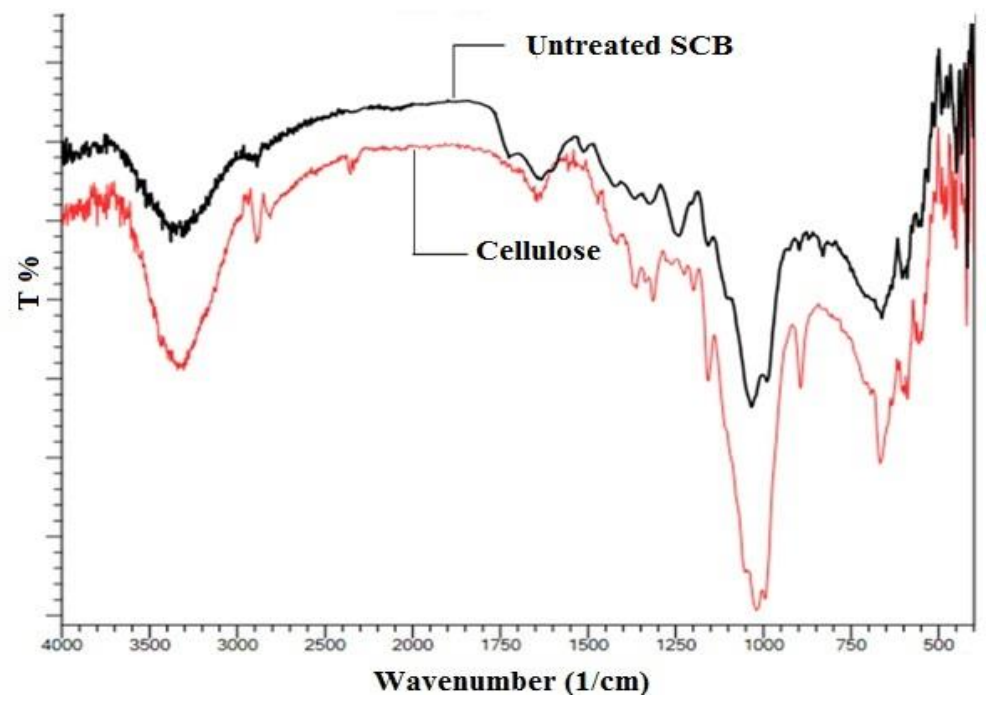

Figure 2. FTIR spectrum of ground untreated SCB and cellulose.

Based on the method described by Rabek (1980), in equation (1), the degree of crystallinity of CNPs was observed to be $88.3 \%$ that proving the exclusion of amorphous properties of cellulose [31]. Similar to this study, the rise in crystalline nature has also been observed by Said Azizi-Samir et al. (2004) [32], Tang et al. (1996) [33], and Mandal and Chakrabarty (2011) [4]. Using the Scherrer equation (2), the crystal size of CNPs was identified to be $30 \mathrm{~nm}$ approximately. Since this material has high crystallinity, it can be concluded that CNPs have great potential to be used as reinforcement agents to produce nanocomposites and for various applications.

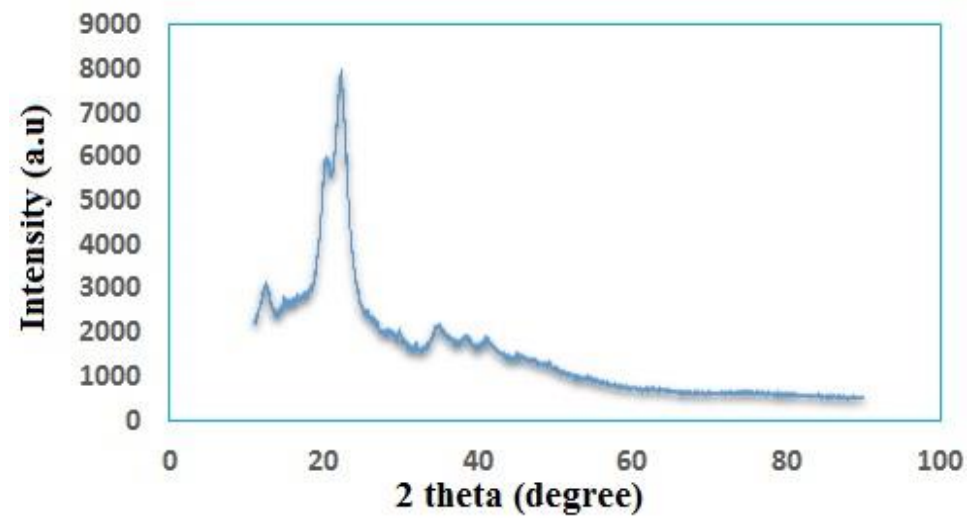

Figure 3. X-ray diffractogram of synthesized CNPs from SCB.

\subsubsection{Scanning electron microscopy (SEM).}

Figure 4 shows the morphology of cellulose nanoparticles prepared from cellulose fiber (Figure $4 \mathrm{a}$ and $\mathrm{b}$ ). After excluding moisture from the nanoparticles obtained, the particles get assembled into fibers of sub-microns and micrometer length [34] because of the rise in $\mathrm{H}$ bonding between -OH groups present on the cellulose surface. The acid-hydrolyzed CNPs showed spherical and rectangular rod-like structures. A similar observation has been described by Ramesh and Radhakrishnan (2019) [13]. 

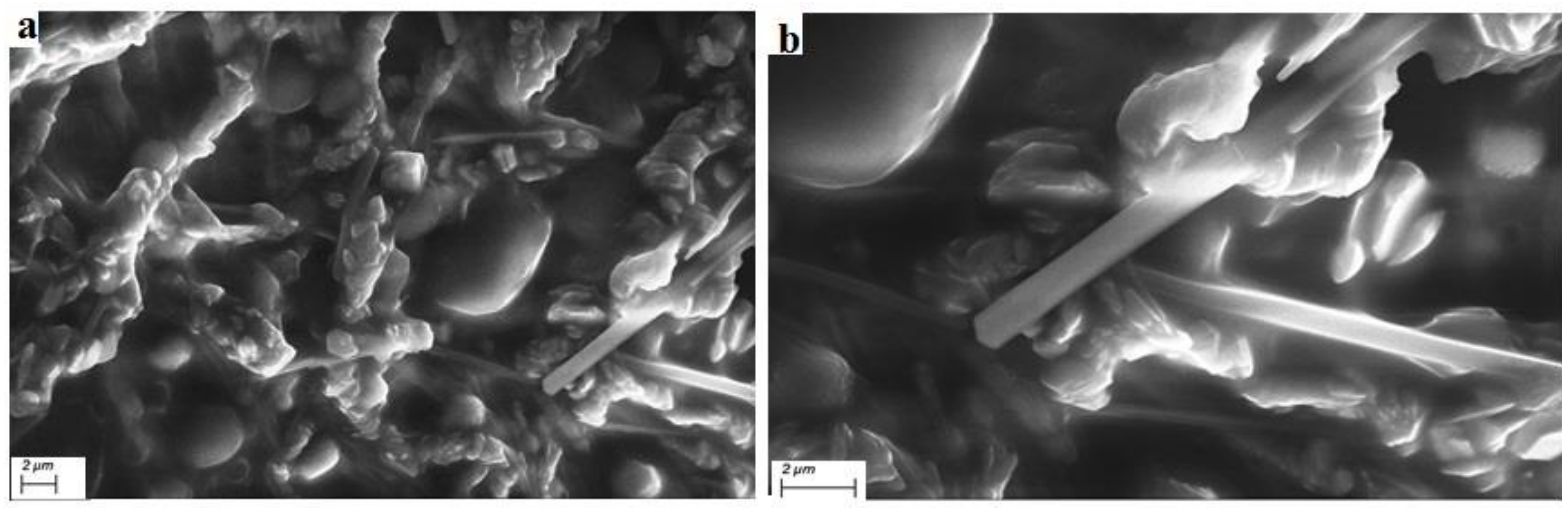

Figure 4. SEM images show the morphological analysis of CNPs from SCB at different magnifications.

3.2.4. Particle size analysis (PSA).

The DLS method was used to analyze the size distribution of the CNPs, synthesized from SCB. As shown in Figure 5, the size distribution signifies that the nanoparticle comprises particles in nanometers. The cellulose nanoparticle diameter was observed to be $189 \mathrm{~nm}$. The smallest size of the particle is approximately $52.10 \mathrm{~nm}$. This intensity distribution depicts that the particle size after the chemical treatment is $90 \%$ lesser than $1056.80 \mathrm{~nm}$, which ascertains that the particle lies in the nanometric range.

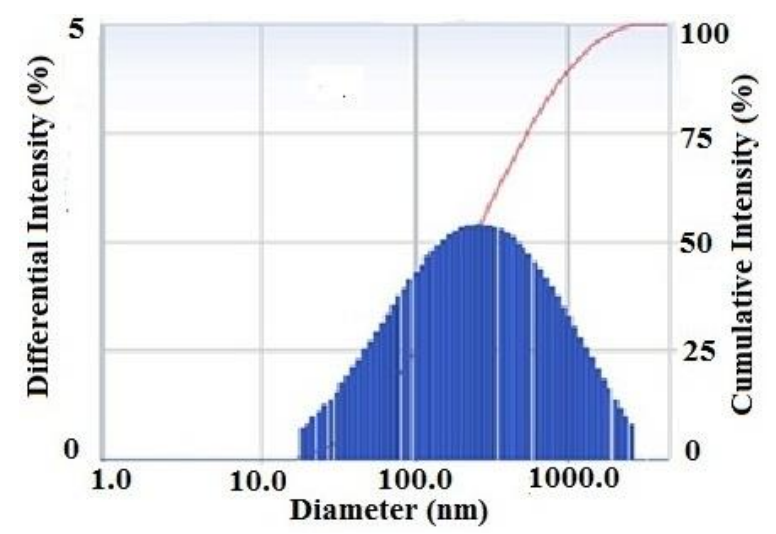

Figure 5. Particle size distribution of CNPs from DLS method.

\section{Conclusions}

This study affirms that cellulose can be acquired from agro-industrial wastes like sugarcane bagasse. The elimination of compounds other than cellulose from SCB was performed effectively using a bleaching agent, sodium chlorite, and an alkali, sodium hydroxide. After hydrolysis using sulfuric acid, further purification of cellulose nanoparticles was done by centrifugation and dialysis. The acid-hydrolyzed cellulose showed a higher degree of crystallinity, which was confirmed by XRD and proves this material can be used as reinforcement agents for the manufacturing of nanocomposites. The obtained SEM images and intensity distribution of CNPs depict that the size of the obtained particle is in nanoscale. As a result, it proves to be valuable in the employment of SCB for CNP preparation and its applications in food packaging film development.

\section{Funding}

This research received no external funding. 


\section{Acknowledgments}

We sincerely thank the management for their support and encouragement throughout the research.

\section{Conflicts of Interest}

The authors declare no conflict of interest.

\section{References}

1. Kumar, A.; Negi, Y.S.; Choudhary, V.; Bhardwaj, N.K. Characterization of cellulose nanocrystals produced by acid-hydrolysis from sugarcane bagasse as agro-waste. Mater. Chem. Phys. 2014, 2, 1-8, http://dx.doi.org/10.12691/jmpc-2-1-1.

2. Mishra, M.K.; Khare, N.; Agrawal, A.B. Bagasse cogeneration in India: status, barriers. IOSR J. Mech. Civ. Eng. 2014, 11, 69-78, http://dx.doi.org/10.9790/1684-11136978.

3. Torgbo, S.; Quan, V.M.; Sukyai, P. Cellulosic value-added products from sugarcane bagasse. Cellulose, 2021, 28, 5219-5240, http://dx.doi.org/10.1007/s10570-021-03918-3.

4. Mokhena, T.C.; Mochane, M.J.; Motaung, T.E.; Linganiso, L.Z.; Thekisoe, O.M.; Songca, S.P. Sugarcane bagasse and cellulose polymer composites. Sugarcane-Technology and Research 2018, 225-40, https://doi.org/10.5772/INTECHOPEN.71497.

5. Paroha, S.; Srivastava, V.; Chaturvedi, N. Sugarcane Bagasse as Dietary Fibre. Indian. J. Pure App. Biosci 2020, 8, 590-597, http://dx.doi.org/10.18782/2582-2845.8442.

6. Sun, J.X.; Sun, X.F.; Zhao, H.; Sun, R.C. Isolation and characterization of cellulose from sugarcane bagasse. Polym. Degrad. Stab. 2004, 84, 331-339, http://dx.doi.org/10.1016/j.polymdegradstab.2004.02.008.

7. Brant, A.J.C.; Naime, N.; Lugão, A.B.; Ponce, P. Cellulose Nanoparticles Extracted from Sugarcane Bagasse and Their Use in Biodegradable Recipients for Improving Physical Properties and Water Barrier of the Latter. Materials Sciences and Applications 2020, 11, 81-133, http://dx.doi.org/10.4236/msa.2020.111007.

8. Mandal, A.; Chakrabarty, D. Isolation of nanocellulose from waste sugarcane bagasse (SCB) and its characterization. Carbohydr. Polym. 2011, 86, 1291-1299, http://dx.doi.org/10.1016/j.carbpol.2011.06.030.

9. Franco, T.S.; de Muniz, G.I.B. Nanocellulose in Food Science and Technology-Potential, Advantages and Gaps of Research. Novel Techniques in Nutrition and Food Science 2018, 1, http://dx.doi.org/10.31031/ntnf.2018.01.000514.

10. Kumar, A.; Negi, Y.S.; Choudhary, V.; Bhardwaj, N. K. Microstructural and mechanical properties of porous biocomposite scaffolds based on polyvinyl alcohol, nano-hydroxyapatite and cellulose nanocrystals. Cellulose 2014, 21, 3409-3426, https://doi.org/10.1007/s10570-014-0339-7.

11. Widiarto, S.; Yuwono, S.D.; Rochliadi, A.; Arcana, I.M. Preparation and characterization of cellulose and nanocellulose from agro-industrial waste-cassava peel. IOP Conference Series: Materials Science and Engineering 2017, 176, 012052, IOP Publishing, https://doi.org/10.1088/1757-899X/176/1/012052.

12. Song, K., Zhu, X.; Zhu, W.; Li, X. Preparation and characterization of cellulose nanocrystal extracted from Calotropis procera biomass. Bioresour. Bioprocess. 2019, 6, 45, https://doi.org/10.1186/s40643-019-0279$\mathrm{z}$.

13. Ramesh, S.; Radhakrishnan, P. Cellulose nanoparticles from agro-industrial waste for the development of active packaging. Appl. Surf. Sci. 2019, 484, 1274-1281, https://doi.org/10.1016/J.APSUSC.2019.04.003.

14. Purkait, B.S.; Ray, D.; Sengupta, S.; Kar, T.; Mohanty, A.; Misra, M. Isolation of cellulose nanoparticles from sesame husk. Ind. Eng. Chem. Res. 2011, 50, 871-876, https://doi.org/10.1021/ie101797d.

15. Trilokesh, C.; Uppuluri, K.B. Isolation and characterization of cellulose nanocrystals from jackfruit peel. Sci Rep 2019, 9, 16709, https://doi.org/10.1038/s41598-019-53412-x.

16. dos Santos, R.M.; Neto, W.P.F.; Silvério, H.A.; Martins, D.F.; Dantas, N.O.; Pasquini, D. Cellulose nanocrystals from pineapple leaf, a new approach for the reuse of this agro-waste. Ind. Crops Prod. 2013, 50, 707-714, http://dx.doi.org/10.1016/j.indcrop.2013.08.049.

17. Reddy, J.P; Rhim, J-W. Isolation and characterization of cellulose nanocrystals from garlic skin. Mater. Lett. 2014, 129, 20-23, http://dx.doi.org/10.1016/j.matlet.2014.05.019. 
18. Wulandari, W.T.; Rochliadi, A.; Arcana, I.M. Nanocellulose prepared by acid hydrolysis of isolated cellulose from sugarcane bagasse. IOP conference series: Materials Science and Engineering 2016, 107, 012045, IOP Publishing, http://dx.doi.org/10.1088/1757-899X/107/1/012045.

19. Plermjai, K.; Boonyarattanakalin, K.; Mekprasart, W.; Pavasupree, S.; Phoohinkong, W.; Pecharapa, W. Extraction and characterization of nanocellulose from sugarcane bagasse by ball-milling-assisted acid hydrolysis. in AIP Conference Proceedings 2018, 2010, 020005, AIP Publishing LLC, http://dx.doi.org/10.1063/1.5053181.

20. Tan, V.Y.; Chou, P.M. Effectiveness of using different mechanical methods for extraction of nanocellulose from kapok husk. AIP Conference Proceedings 2020, 2233, 020027, AIP Publishing LLC, http://dx.doi.org/10.1063/5.0001967.

21. Jancy, S., Shruthy, R.; Preetha, R. Fabrication of packaging film reinforced with cellulose nanoparticles synthesised from jack fruit non-edible part using response surface methodology. Int. J. Biol. Macromol. 2020, 142, 63-72, http://dx.doi.org/10.1016/j.ijbiomac.2019.09.066.

22. Updegraff, D.M. Semimicro determination of cellulose inbiological materials. Anal. Biochem. 1969, 32, 420424, http://dx.doi.org/10.1016/S0003-2697(69)80009-6.

23. Wyman, C.E.; Decker, S.R.; Himmel, M.E.; Brady, J.W.; Skopec, C.E.; Viikari, L. Hydrolysis of cellulose and hemicellulose. Polysaccharides: Structural diversity and functional versatility 2005, 995-1034, 2-nd ed., Marcel Dekker, Inc.

24. Costa, L.A.S.; Assis, D.J.; Gomes, G.V.P.; da Silva, J.B.A,; Fonseca, A.F.; Druzian, J.I. Extraction and characterization of cellulose nanocrystals from corn stover. Materialstoday: Proceedings 2015, 2, 287-294, http://dx.doi.org/10.1016/j.matpr.2015.04.045.

25. de Oliveira, J.P.; Bruni, G.P.; El Halal, S.L.M.; Bertoldi, F.C.; Dias, A.R.G.; da Rosa Zavareze, E. Cellulose nanocrystals from rice and oat husks and their application in aerogels for food packaging. Int. J. Biol. Macromol. 2019, 124, 175-184, http://dx.doi.org/10.1016/j.ijbiomac.2018.11.205.

26. Meyabadi, T.F.; Dadashian, F.; Sadeghi, G.M.M.; Asl, H.E.Z. Spherical cellulose nanoparticles preparation from waste cotton using a green method. Powder Technol. 2014, 261, 232-240, http://dx.doi.org/10.1016/j.powtec.2014.04.039.

27. Sun, R.; Lawther, J.M; Banks, W.B. The fractional composition of polysaccharides and lignin in alkaline pretreated and steam pressure treated wheat straw. The Chemistry and Processing of Wood and Plant Fibrous Material, Cellucon '94 Proceedings 1996, 291-312, Woodhead Publishing, http://dx.doi.org/10.1533/9781845698690.291.

28. Yue, Y.; Han, J.; Han, G.; Aita, G.M.; Wu, Q. Cellulose fibers isolated from energycane bagasse using alkaline and sodium chlorite treatments: Structural, chemical and thermal properties. Ind. Crops Prod. 2015, 76, 355-363, http://dx.doi.org/10.1016/j.indcrop.2015.07.006.

29. Johar, N.; Ahmad, I.; Dufresne, A. Extraction, preparation and characterization of cellulose fibres and nanocrystals from rice husk. Ind. Crops Prod. 2012, 37, 93-99, http://dx.doi.org/10.1016/j.indcrop.2011.12.016.

30. Evans, S.K.; Wesley, O.N.; Nathan, O.; Moloto, M.J. Chemically purified cellulose and its nanocrystals from sugarcane baggase: isolation and characterization. Heliyon 2019, 5, e02635, http://dx.doi.org/10.1016/j.heliyon.2019.e02635.

31. Phanthong, P.; Reubroycharoen, P.; Hao, X.; Xu, G.; Abudula, A.; Guan, G. Nanocellulose: Extraction and application. Carbon Resources Conversion 2018, 1, 32-43, http://dx.doi.org/10.1016/j.crcon.2018.05.004.

32. Saïd Azizi Samir, M.A.; Alloin, F.; Paillet, M.; Dufresne, A. Tangling effect in fibrillated cellulose reinforced nanocomposites. Macromol 2004, 37, 4313-4316, http://dx.doi.org/10.1021/ma035939u.

33. Tang, L.G.; Hon, D.N.S.; Pan, S.H.; Zhu, Y.Q.; Wang, Z.; Wang, Z.Z. Evaluation of microcrystalline cellulose. I. Changes in ultrastructural characteristics during preliminary acid hydrolysis. J. Appl. Polym. Sci. 1996, 59, 483-488, https://doi.org/10.1002/\%28SICI\%2910974628\%2819960118\%2959\%3A3\%3C483\%3A\%3AAID-APP13\%3E3.0.CO\%3B2-V.

34. Fortunati, E.; Puglia, D.; Monti, M.; Peponi, L.; Santulli, C.; Kenny, J.M.; Torre, L. Extraction of cellulose nanocrystals from Phormium tenax fibres. J. Polym. Environ. 2013, 21, 319-328, http://dx.doi.org/10.1007/s10924-012-0543-1. 